\title{
Stellar populations of bulges in galaxies with a low surface-brightness disc
}

\author{
L. Morelli $\mathbf{i}^{1,2 \dagger}$ E. M. Corsini $\mathbf{i}^{1,2}$, A. Pizzella ${ }^{1,2}$, E. Dalla Bontà ${ }^{1,2}$, \\ L. Coccato ${ }^{3}$, J. Méndez-Abreu ${ }^{4,5}$ and M. Cesetti ${ }^{1,2}$ \\ ${ }^{1}$ Dipartimento di Fisica e Astronomia, Università di Padova, vicolo dell'Osservatorio 3, I-35122 \\ Padova, Italy \\ ${ }^{2}$ INAF-Osservatorio Astronomico di Padova, vicolo dell'Osservatorio 5, I-35122 Padova, Italy \\ ${ }^{3}$ European Southern Observatory, Karl-Schwarzschild-Straße 2, D-85748 Garching bei \\ München, Germany \\ ${ }^{4}$ Instituto Astrofísico de Canarias, C/ Vía Láctea s/n, E-38200 La Laguna, Spain \\ ${ }^{5}$ Departamento de Astrofísica, Universidad de La Laguna, Universidad de La Laguna, C/ \\ Astrofísico Francisco Sánchez, E-38205 La Laguna, Spain
}

\begin{abstract}
The radial profiles of the $\mathrm{H}_{\beta}, \mathrm{Mg}$, and Fe line-strength indices are presented for a sample of eight spiral galaxies with a low surface-brightness stellar disc and a bulge. The correlations between the central values of the line-strength indices and velocity dispersion are consistent to those known for early-type galaxies and bulges of high surface-brightness galaxies. The age, metallicity, and $\alpha / \mathrm{Fe}$ enhancement of the stellar populations in the bulge-dominated region are obtained using stellar population models with variable element abundance ratios. Almost all the sample bulges are characterized by a young stellar population, on-going star formation, and a solar $\alpha /$ Fe enhancement. Their metallicity spans from high to sub-solar values. No significant gradient in age and $\alpha / \mathrm{Fe}$ enhancement is measured, whereas only in a few cases a negative metallicity gradient is found. These properties suggest that a pure dissipative collapse is not able to explain formation of all the sample bulges and that other phenomena, like mergers or acquisition events, need to be invoked. Such a picture is also supported by the lack of a correlation between the central value and gradient of the metallicity in bulges with very low metallicity. The stellar populations of the bulges hosted by low surface-brightness discs share many properties with those of high surface-brightness galaxies. Therefore, they are likely to have common formation scenarios and evolution histories. A strong interplay between bulges and discs is ruled out by the fact that in spite of being hosted by discs with extremely different properties, the bulges of low and high surface-brightness discs are remarkably similar.
\end{abstract}

Keywords. galaxies : abundances - galaxies : bulges - galaxies : evolution - galaxies : stellar content - galaxies : formation - galaxies : Kinemaitics and Dynamics 